PROCEEDINGS OF THE

AMERICAN MATHEMATICAL SOCIETY

Volume 137, Number 3, March 2009, Pages 965-970

S 0002-9939(08)09635-4

Article electronically published on September 11, 2008

\title{
ON SPACES OF OPERATORS ON $C(Q)$ SPACES ( $Q$ COUNTABLE METRIC SPACE)
}

\author{
CHRISTIAN SAMUEL
}

(Communicated by Nigel J. Kalton)

\begin{abstract}
In this paper we study spaces of nuclear operators $\mathcal{N}(C(Q))$ and spaces of compact operators $\mathcal{K}(C(Q))$ on spaces of continuous functions $C(Q)$, where $Q$ is a countable compact metric space, in connection with the C. Bessaga and A. Pełczyński isomorphic classification of these spaces.

We show that the spaces $\mathcal{K}(C(Q))$ [resp. $\mathcal{N}(C(Q))$ ] and $\mathcal{K}\left(C\left(Q^{\prime}\right)\right)$ [resp. $\left.\mathcal{N}\left(C\left(Q^{\prime}\right)\right)\right]$ are isomorphic if, and only if, $C(Q)$ and $C\left(Q^{\prime}\right)$ are isomorphic. We show also that $\mathcal{N}(C(Q))$ is not isomorphic to a subspace of $\mathcal{K}(C(Q))$.
\end{abstract}

\section{NotATion AND TERMinOLOGY}

Throughout this paper, the symbols $E, F, X, Y, \ldots$ denote Banach spaces. $B_{E}$ denotes the closed unit ball of $E$. "Subspace" means closed linear subspace. "Operator" means "bounded linear operator".

In the sequel we denote $\alpha, \beta, \gamma, \ldots$ ordinal numbers, $\omega$ denotes the first infinite ordinal and $\omega_{1}$ the first uncountable ordinal. Let $\alpha<\beta$ be ordinals; then $\langle\alpha, \beta\rangle$ denotes the interval $\{\gamma ; \alpha \leq \gamma \leq \beta\}$ and $\langle\alpha, \beta)$ denotes the interval $\{\gamma ; \alpha \leq \gamma<$ $\beta\}$ endowed with the order topology. It is a well-known theorem of Mazurkiewicz and Sierpiński, cf. [5], that every countable compact metric space is homeomorphic to an interval $\langle 1, \alpha\rangle$ with $\omega \leq \alpha<\omega_{1}$.

$C(\alpha)$ denotes the space of all continuous scalar-valued functions defined on $\langle 1, \alpha\rangle$ with the norm $\|x\|=\sup _{\gamma \in\langle 1, \alpha\rangle}|x(\gamma)|$ and $C_{0}(\alpha)$ is the subspace $\{x \in C(\alpha) ; x(\alpha)=$ $0\}$ of $C(\alpha)$. The space of all continuous $E$-valued functions defined on $\langle 1, \alpha\rangle$ with the norm $\|x\|=\sup _{\gamma \in\langle 1, \alpha\rangle}\|x(\gamma)\|$ is denoted $C(\alpha, E)$ and $C_{0}(\alpha, E)$ is the subspace $\{x \in C(\alpha, E) ; x(\alpha)=0\}$. The spaces $C(\alpha, E)$ and $C_{0}(\alpha, E)$ are isomorphic for all $\alpha \in\left\langle\omega, \omega_{1}\right\rangle ;$ cf. [1].

The injective norm on $E \otimes F$, denoted by \|\|$_{\varepsilon}$, is given by

$$
\left\|\sum_{i=1}^{n} x_{i} \otimes y_{i}\right\|_{\varepsilon}=\sup \left\{\left|\sum_{i=1}^{n} x^{*}\left(x_{i}\right) y^{*}\left(y_{i}\right)\right| ; x^{*} \in B_{E^{*}}, y^{*} \in B_{F^{*}}\right\} .
$$

The completion of $\left(E \otimes F,\|\|_{\varepsilon}\right)$ is denoted by $E \widehat{\widehat{\otimes}} F$.

Received by the editors February 19, 2008.

2000 Mathematics Subject Classification. Primary 46B03, 46B25; Secondary 47B10.

Key words and phrases. Isomorphic classification of spaces of continuous functions, nuclear operators, compact operators. 
The projective norm on $E \otimes F$, denoted by \|\|$_{\pi}$, is given by

$$
\|u\|_{\pi}=\sup \{|\varphi(u)| ; \varphi \text { bounded bilinear form on } E \times F,\|\varphi\| \leq 1\} .
$$

The completion of $\left(E \otimes F,\|\|_{\pi}\right)$ is denoted by $E \widehat{\otimes} F$.

It is well known that (cf. [2], [3]):

i) given $(\Omega, \mathcal{S}, \mu)$ a measure space, the space $L^{1}(\mu) \widehat{\otimes} E$ may be isometrically identified with the space $L^{1}(\mu, E)$ of Bochner-integrable "functions" from $\Omega$ to $E$,

ii) given $K$ a topological compact space, the space $C(K) \widehat{\widehat{\otimes}} E$ is isometrically isomorphic to the space $C(K, E)$ of all continuous $E$-valued functions defined on $K$.

An operator $T: E \rightarrow F$ is called nuclear if it can be written as the sum of an absolutely converging series of rank-one operators, i.e., if there are sequences $\left(x_{n}^{*}\right)_{n}$ in $E^{*}$ and $\left(y_{n}\right)_{n}$ in $F$ with $\sum_{n}\left\|x_{n}^{*}\right\|\left\|y_{n}\right\|<+\infty$ and such that $T(x)=\sum_{n} x_{n}^{*}(x) y_{n}$ for all $x \in E$. Such a series $\sum_{n} x_{n}^{*} \otimes y_{n}$ is called a nuclear representation of $T$; the infimum

$$
\|T\|_{\mathcal{N}}=\inf \left\{\sum_{n}\left\|x_{n}^{*}\right\|\left\|y_{n}\right\| ; \sum_{n} x_{n}^{*} \otimes y_{n} \text { nuclear representation of } T\right\}
$$

is called the nuclear norm of $T$. We denote by $\mathcal{N}(E, F)$ the space of nuclear operators from $E$ to $F, \mathcal{N}(E)=\mathcal{N}(E, E)$. The space $\left(\mathcal{N}(E, F),\|\|_{\mathcal{N}}\right)$ is a Banach space. Also, $\mathcal{L}(X, Y)[$ resp. $\mathcal{K}(X, Y)]$ denotes the space of operators [resp. of compact operators] from $X$ to $Y, \mathcal{L}(X)=\mathcal{L}(X, X), \mathcal{K}(X)=\mathcal{K}(X, X)$.

There exists a closed connection between compact or nuclear operators and tensor products. If $E^{*}$ or $F$ has the approximation property, then (cf. [2, 3]):

i) the canonical map from $E^{*} \widehat{\otimes} F$ into $\mathcal{L}(E, F)$ is an isomorphism onto $\mathcal{N}(E, F)$,

ii) the canonical map from $E^{*} \widehat{\widehat{\otimes}} F$ into $\mathcal{L}(E, F)$ is an isomorphism onto $\mathcal{K}(E, F)$. The author is grateful to the referee for helpful comments.

\section{The SPACES $\mathcal{N}(C(Q))$ and $\mathcal{K}\left(C\left(Q^{\prime}\right)\right)$}

W.B. Johnson has shown in 4 that if either $X$ or $Y$ has a local unconditional structure, then $\mathcal{N}(X, Y)$ is a proper subset of $\mathcal{K}(X, Y)$. In this section we shall show that no subspace of $\mathcal{K}\left(C\left(Q^{\prime}\right)\right)$ is isomorphic to $\mathcal{N}(C(Q))$ whatever the countable metric compact spaces $Q$ and $Q^{\prime}$ are.

Lemma 2.1. Let $\alpha$ be a countable ordinal $\left(\omega \leq \alpha<\omega_{1}\right)$ and let $E$ be a Banach space which does not contain a subspace isomorphic to $c_{0}$ and such that $E \times E$ is isomorphic to a subspace of $E$. Then every subspace of $C(\alpha, E)$ which does not contain a subspace isomorphic to $c_{0}$ is isomorphic to a subspace of $E$.

Proof. We prove the lemma by a transfinite induction on $\alpha$. The proof of the initial case $\alpha=\omega$ is essentially the same as the proof of the case $\omega<\alpha$ where $\alpha$ is a limit ordinal. Suppose that $\alpha$ is a limit ordinal which satisfies $\omega<\alpha<\omega_{1}$ and that, for every $\beta \in\langle\omega, \alpha)$, every subspace of $C(\beta, E)$ which does not contain a subspace isomorphic to $c_{0}$ is isomorphic to a subspace of $E$.

Consider a subspace $X$ of $C_{0}(\alpha, E)$ which does not contain a subspace isomorphic to $c_{0}$. 
Let $\omega \leq \alpha_{1}<\alpha_{2}<\ldots<\alpha_{k}<\ldots<\alpha$ be a strictly increasing sequence of ordinal numbers converging to $\alpha$. If there exists an integer $k_{0}$ such that $X$ is isomorphic to a subspace of $C\left(\alpha_{k_{0}}, E\right)$, the conclusion will then follow from the induction hypothesis.

Assume on the contrary that, for every integer $k, X$ is not isomorphic to a subspace of $C\left(\alpha_{k}, E\right)$. Let $\left.\varepsilon \in\right] 0,1[$. By a standard procedure using the operators

$$
\pi_{k}: C_{0}(\alpha, E) \rightarrow C\left(\alpha_{k}, E\right)
$$

defined, for every integer $k$, by $\pi_{k}(f)=f_{\mid\left\langle 1, \alpha_{k}\right\rangle}$, we can find a strictly increasing sequence of integers $\left(k_{l}\right)_{l \geq 1}$ and a normalized sequence $\left(f_{l}\right)_{l \geq 1}$ of $X$ such that, for every integer $l \geq 2$, we have $\left\|\pi_{k_{l-1}}\left(f_{l}\right)\right\| \leq \frac{\varepsilon}{2^{l}}$ and, for every integer $l \geq 1$ and for every ordinal $\gamma>\alpha_{k_{l}}$, we have $\left\|f_{l}(\gamma)\right\| \leq \frac{\varepsilon^{2}}{2^{l}}$.

It is easy to show that for every integer $l_{0}$ and for every finite scalar sequence $a_{1}, \ldots, a_{l_{0}}$ we have

$$
(1-\varepsilon) \max _{1 \leq l \leq l_{0}}\left|a_{l}\right| \leq\left\|\sum_{l=1}^{l_{0}} a_{l} f_{l}\right\| \leq(1+\varepsilon) \max _{1 \leq l \leq l_{0}}\left|a_{l}\right| .
$$

So $\left(f_{l}\right)_{l \geq 1}$ is a normalized basic sequence of $\mathrm{X}$ equivalent to the canonical basis of $c_{0}$. The assumption that, for every integer $k, X$ is not isomorphic to a subspace of $C\left(\alpha_{k}, E\right)$ leads to a contradiction.

In the case $\alpha=\omega$ we have to observe that a subspace $X$ of $C_{0}(\omega, E)$ which does not contain a subspace isomorphic to $c_{0}$ is isomorphic to a subspace of $E^{n}$ for some integer $n$, so $X$ is isomorphic to a subspace of $E$.

The case where $\alpha$ is a successor ordinal is obvious.

The proof of the following lemma is straightforward.

Lemma 2.2. Every infinite dimensional subspace of $\left(\ell_{\infty}^{1} \oplus \ldots \oplus \ell_{\infty}^{n} \oplus \ldots\right)_{\ell_{1}}$ contains a subspace isomorphic to $\ell_{1}$.

Theorem 2.3. Let $\alpha, \beta$ be two countable ordinals; then $\mathcal{N}(C(\alpha))$ is not isomorphic to a subspace of $\mathcal{K}(C(\beta))$.

Proof. Suppose that there exists an isomorphism $T$ from $\mathcal{N}\left(C_{0}(\alpha)\right)$ onto a subspace of $\mathcal{K}\left(C_{0}(\beta)\right)$. We know that $\mathcal{N}\left(C_{0}(\alpha)\right)$ is isometrically isomorphic to $\ell_{1} \widehat{\otimes} C_{0}(\alpha)=$ $\ell_{1}\left(\mathbb{N}, C_{0}(\alpha)\right)$ and $\mathcal{K}\left(C_{0}(\beta)\right)$ is isometrically isomorphic to $\ell_{1} \widehat{\widehat{\otimes}} C_{0}(\beta)=C_{0}\left(\beta, \ell_{1}\right)$. Let, for every integer $n, X_{n}$ be a subspace of $C_{0}(\alpha)$ isometric to $\ell_{\infty}^{n}$. By Lemma 2.2. the subspace $X=\left(X_{1} \oplus \cdots \oplus X_{n} \oplus \cdots\right) \ell_{1}$ of $\mathcal{N}\left(C_{0}(\alpha)\right)$ does not contain a subspace isomorphic to $c_{0}$, so, by Lemma 2.1 with $E=\ell_{1}$, the subspace $T(X)$ of $C_{0}\left(\beta, \ell_{1}\right)$ is isomorphic to a subspace of $\ell_{1}$. This means that $c_{0}$ is finitely representable in $\ell_{1}$, which is, of course, false and ends the proof.

Using the same arguments we can prove

Theorem 2.4. Let $Q_{1}, Q_{2}, Q_{3}$ and $Q_{4}$ be four countable compact metric spaces. Then $\mathcal{N}\left(C\left(Q_{1}\right), C\left(Q_{2}\right)\right)$ is not isomorphic to a subspace of $\mathcal{K}\left(C\left(Q_{3}\right), C\left(Q_{4}\right)\right)$. 


\section{SPACES OF COMPACT OPERATORS}

The isomorphic classification of $C(Q)$ spaces with $Q$ a countable compact metric space is due to C. Bessaga and A. Pełczyński [1. Let $\omega \leq \alpha \leq \beta<\omega_{1}$ be countable ordinal numbers. Then the spaces $C(\alpha)$ and $C(\beta)$ are isomorphic if, and only if, $\beta<\alpha^{\omega}$. Thus, under the conditions $\omega \leq \alpha \leq \beta<\alpha^{\omega}<\omega_{1}$, the spaces $\mathcal{K}(C(\alpha))$ and $\mathcal{K}(C(\beta))$ are isomorphic. Conversely we shall show that, if $\mathcal{K}(C(\alpha))$ and $\mathcal{K}(C(\beta))$ are isomorphic, then the spaces $C(\alpha)$ and $C(\beta)$ are also isomorphic.

Lemma 3.1. Let $\alpha$ be a countable ordinal $\left(\omega \leq \alpha<\omega_{1}\right)$ and let $E$ be a Banach space. If, for every ordinal $\gamma<\alpha$, the space $C_{0}(\alpha)$ is not isomorphic to a subspace of $C_{0}(\gamma, E)$, then the space $C_{0}\left(\alpha^{\omega}\right)$ is not isomorphic to a subspace of $C_{0}(\alpha, E)$.

Proof. We use ideas of the proof of Lemma 3 in [1. Suppose that $\alpha$ is a countable ordinal such that for every ordinal $\gamma<\alpha$, the space $C_{0}(\alpha)$ is not isomorphic to a subspace of $C_{0}(\gamma, E)$ and $C_{0}\left(\alpha^{\omega}\right)$ is isomorphic to a subspace of $C_{0}(\alpha, E)$; we shall show that this assumption leads to a contradiction. Let $T$ be an operator from $C_{0}\left(\alpha^{\omega}\right)$ into $C_{0}(\alpha, E)$ and $b$ be a real number such that, for every $f \in C_{0}\left(\alpha^{\omega}\right)$, we have

$$
\|f\| \leq\|T(f)\| \leq b\|f\| .
$$

Now we fix an integer $N$ and $\varepsilon \in] 0,1\left[\right.$ such that $b<N$ and $\frac{N}{N+1} \leq \frac{1-\varepsilon}{1+\varepsilon}$.

Let $f_{0}$ be the function identically equal to 1 on $\left\langle 1, \alpha^{N}\right\rangle$ and equal to 0 on $\left\langle\alpha^{N}+\right.$ $\left.1, \alpha^{\omega}\right\rangle$. There exists an ordinal $\gamma_{1}<\alpha$ such that $\left\|T\left(f_{0}\right)(\gamma)\right\| \leq \varepsilon$ for every $\gamma_{1}<\gamma \leq$ $\alpha$. Let $\Delta_{\beta}^{1}=\left\langle\alpha^{N-1} \beta+1, \alpha^{N-1}(\beta+1)\right\rangle$ for $0 \leq \beta<\alpha$ and

$E_{1}=\left\{f \in C_{0}\left(\alpha^{\omega}\right) ; \forall \gamma>\alpha^{N}, f(\gamma)=0\right.$ and $\forall 0 \leq \beta<\alpha, f$ is constant on $\left.\Delta_{\beta}^{1}\right\}$.

It is obvious that $E_{1}$ is isomorphic to $C_{0}(\alpha)$. There is no subspace of $C_{0}\left(\gamma_{1}, \ell_{1}\right)$ isomorphic to $C_{0}(\alpha)$, so there exists $f_{1} \in E_{1}$ such that $\left\|f_{1}\right\|=1$ and, for every $\gamma \leq \gamma_{1}$, we have $\left\|T\left(f_{1}\right)(\gamma)\right\| \leq \varepsilon$. We may change $f_{1}$ to $-f_{1}$ and suppose that there exists $\beta_{1} \in\langle 0, \alpha)$ such that, for every $\lambda \in \Delta_{\beta_{1}}^{1}$, we have $1-\varepsilon \leq f_{1}(\lambda) \leq 1$. We have $T\left(f_{1}\right) \in C_{0}\left(\alpha, \ell_{1}\right)$, so we can find $\gamma_{2} \in\left(\gamma_{1}, \alpha\right)$ such that $\left\|T\left(f_{1}\right)(\gamma)\right\| \leq \varepsilon$ for every $\gamma_{2}<\gamma<\alpha$. This ends the first step of the proof.

For the second step, let

$$
\Delta_{\beta}^{2}=\left\langle\alpha^{N-1} \beta_{1}+\alpha^{N-2} \beta+1, \alpha^{N-1} \beta_{1}+\alpha^{N-2}(\beta+1)\right\rangle
$$

for $0 \leq \beta<\alpha$ and let

$$
E_{2}=\left\{f \in C_{0}\left(\alpha^{\omega}\right) ; \forall \gamma \notin \Delta_{\beta_{1}}^{1}, f(\gamma)=0 \text { and } \forall 0 \leq \beta<\alpha, f \text { is constant on } \Delta_{\beta}^{2}\right\} .
$$

The subspace $E_{2}$ of $C_{0}\left(\alpha^{\omega}\right)$ is isomorphic to $C_{0}(\alpha)$. In the same way as in the previous case, we can find $f_{2} \in E_{2},\left\|f_{2}\right\|=1$ and two ordinal numbers $0 \leq \beta_{2}<\alpha$, $\gamma_{2}<\gamma_{3}<\alpha$ such that $\left\|T\left(f_{2}\right)(\gamma)\right\| \leq \varepsilon$ for all $\gamma \leq \gamma_{2},\left\|T\left(f_{2}\right)(\gamma)\right\| \leq \varepsilon$ for all $\gamma>\gamma_{3}$ and $1-\varepsilon \leq f_{2}(\gamma) \leq 1$ for every $\gamma \in \Delta_{\beta_{2}}^{2}$. Repeating this procedure $N$ times we shall find $f_{0}, \ldots, f_{N} \in C_{0}\left(\alpha^{\omega}\right)$ and $\gamma_{1}<\gamma_{2}<\cdots<\gamma_{N}<\alpha$ such that :

- $\left\|f_{0}\right\|=\ldots=\left\|f_{N}\right\|=1$,

- $\emptyset \neq f_{N}^{-1}([1-\varepsilon, 1]) \subset f_{N-1}^{-1}([1-\varepsilon, 1]) \subset \cdots \subset f_{1}^{-1}([1-\varepsilon, 1]) \subset\left\langle 1, \alpha^{N}\right\rangle=$ $f_{0}^{-1}(1)$

- $\forall 1 \leq k \leq N, \forall \gamma \leq \gamma_{k}$ we have $\left\|T\left(f_{k}\right)(\gamma)\right\| \leq \varepsilon$,

- $\forall 1 \leq k \leq N, \forall \gamma>\gamma_{k}$ we have $\left\|T\left(f_{k-1}\right)(\gamma)\right\| \leq \varepsilon$. 
It is obvious that $(N+1)(1-\varepsilon) \leq\left\|f_{0}+\cdots+f_{N}\right\|$ and $\left\|T\left(f_{0}\right)+\cdots+T\left(f_{N}\right)\right\| \leq$ $b+N \varepsilon$; hence $(N+1)(1-\varepsilon) \leq b+N \varepsilon$, and so $N \leq b$. We obtain a contradiction with $b<N$, and so the conclusion of the lemma holds.

Theorem 3.2. Let $\omega \leq \alpha \leq \beta<\omega_{1}$ be two countable infinite ordinal numbers and let $E$ be a Banach space which does not contain a subspace isomorphic to $c_{0}$. Then $C(\beta)$ is isomorphic to a subspace of $C(\alpha, E)$ if, and only if, $C(\beta)$ is isomorphic to a subspace of $C(\alpha)$.

Proof. It is obvious that if $C(\beta)$ is isomorphic to a subspace of $C(\alpha)$, then $C(\beta)$ is isomorphic to a subspace of $C(\alpha, E)$.

For the converse we introduce two sets of ordinal numbers:

$I_{1}=\left\{\alpha \in\left\langle\omega, \omega_{1}\right) ; \forall \gamma<\alpha, C(\alpha)\right.$ is not isomorphic to a subspace of $\left.C(\gamma)\right\}$, $I_{2}=\left\{\alpha \in\left\langle\omega, \omega_{1}\right) ; \forall \gamma<\alpha, C(\alpha)\right.$ is not isomorphic to a subspace of $\left.C(\gamma, E)\right\}$.

We shall prove that $I_{1}=I_{2}$. It is obvious that $I_{2} \subset I_{1}$. The space $E$ does not contain a subspace isomorphic to $c_{0}$, so, for every integer $n$, the space $E^{n}$ does not contain a subspace isomorphic to $c_{0}$; therefore $\omega \in I_{2}$. Now, suppose that $I_{2}$ is a proper subset of $I_{1}$. Let $\alpha_{1}$ be the least element of $I_{1} \backslash I_{2}$. We have $\omega<\alpha_{1}$ and for every $\beta \in I_{1}, \beta<\alpha_{1}$ implies $\beta \in I_{2}$. The ordinal $\alpha_{1} \notin I_{2}$, so there exists an ordinal $\gamma_{1}<\alpha_{1}$ be such that $C\left(\alpha_{1}\right)$ is isomorphic to a subspace of $C\left(\gamma_{1}, E\right)$. Let

$$
\alpha_{2}=\min \left\{\gamma<\alpha_{1} ; C\left(\alpha_{1}\right) \text { is isomorphic to a subspace of } C(\gamma, E)\right\} \text {. }
$$

We have $\alpha_{2} \leq \gamma_{1}$ and $C\left(\alpha_{1}\right)$ is isomorphic to a subspace of $C\left(\alpha_{2}, E\right)$. Let us show that $\alpha_{2} \in I_{1}$. If this is not the case, there exists an ordinal $\gamma_{2}<\alpha_{2}$ such that $C\left(\alpha_{2}\right)$ is isomorphic to a subspace of $C\left(\gamma_{2}\right)$. Therefore $C\left(\alpha_{2}, E\right)$ is isomorphic to a subspace of $C\left(\gamma_{2}, E\right)$ and so $C\left(\alpha_{1}\right)$ is isomorphic to a subspace of $C\left(\gamma_{2}, E\right)$ with $\gamma_{2}<\alpha_{2}$, in contradiction with the definition of $\alpha_{2}$. We have $\alpha_{2} \in I_{1}$ and $\alpha_{2}<\alpha_{1}$, so $\alpha_{2} \in I_{2}$. By Lemma 3.1, the space $C\left(\alpha_{2}^{\omega}\right)$ is not isomorphic to a subspace of $C\left(\alpha_{2}, E\right)$. We have $\alpha_{1} \in I_{1}$ and $\alpha_{2}<\alpha_{1}$, so, by the result of C. Bessaga and A. Pełczyński [1] the space $C\left(\alpha_{2}^{\omega}\right)$ is isomorphic to a subspace of $C\left(\alpha_{1}\right)$. Therefore $C\left(\alpha_{1}\right)$ is not isomorphic to a subspace of $C\left(\alpha_{2}, E\right)$ in contradiction with the definition of $\alpha_{2}$. Hence $I_{1}=I_{2}$ and the converse is proved.

The following theorem is a straightforward consequence of the previous one.

Theorem 3.3. Let $\omega \leq \alpha \leq \beta<\omega_{1}$ be two countable infinite ordinal numbers. Then $\mathcal{K}(C(\alpha))$ is isomorphic to $\mathcal{K}(C(\beta))$ if, and only if, $\beta<\alpha^{\omega}$.

\section{SPACES OF NUCLEAR OPERATORS}

Under the conditions $\omega \leq \alpha \leq \beta<\alpha^{\omega}<\omega_{1}$ the spaces $\mathcal{N}(C(\alpha))$ and $\mathcal{N}(C(\beta))$ are isomorphic. We shall show conversely that if $\mathcal{N}(C(\alpha))$ and $\mathcal{N}(C(\beta))$ are isomorphic, then $C(\alpha)$ and $C(\beta)$ are also isomorphic.

Lemma 4.1. Let $\alpha \in\left\langle\omega, \omega_{1}\right)$ and let $E$ be a subspace of $\ell_{1}(\mathbb{N}, C(\alpha))$ which is not isomorphic to a subspace of $C(\alpha)$. Then there is a subspace of $E$ which is isomorphic to $\ell_{1}$.

Proof. For every integer $k$, let $\pi_{k}$ be the operator on $\ell_{1}(\mathbb{N}, C(\alpha))$ defined by $\pi_{k}(x)=$ $\left(x_{1}, \ldots, x_{k}, 0,0 \ldots\right)$ for every $x=\left(x_{i}\right)_{i \geq 1} \in \ell_{1}(\mathbb{N}, C(\alpha))$. Let $\left.\varepsilon \in\right] 0,1 / 2[$. Using a standard trick we can find a normalized sequence $\left(X_{n}\right)_{n \geq 1}$ of $E$ and a strictly increasing sequence $\left(k_{n}\right)_{n \geq 1}$ of integers such that $\left\|X_{1}-\pi_{k_{1}}\left(X_{1}\right)\right\| \leq \varepsilon$ and 
$\left\|X_{n}-\left(\pi_{k_{n}}-\pi_{k_{n-1}}\right)\left(X_{n}\right)\right\| \leq \varepsilon$ for every integer $n \geq 2$. Let $Y_{1}=\pi_{k_{1}}\left(X_{1}\right)$ and $Y_{n}=\left(\pi_{k_{n}}-\pi_{k_{n-1}}\right)\left(X_{n}\right)$ for $n=2,3, \ldots$ It is obvious that for every integer $n \geq 1$ and for every finite sequence $\left(a_{i}\right)_{1 \leq i \leq n}$ of real numbers we have

$$
(1-\varepsilon) \sum_{i=1}^{n}\left|a_{i}\right| \leq\left\|\sum_{i=1}^{n} a_{i} Y_{i}\right\| \leq \sum_{i=1}^{n}\left|a_{i}\right|
$$

and

so

$$
\left\|\sum_{i=1}^{n} a_{i} Y_{i}-\sum_{i=1}^{n} a_{i} X_{i}\right\| \leq \varepsilon \sum_{i=1}^{n}\left|a_{i}\right|
$$

$$
(1-2 \varepsilon) \sum_{i=1}^{n}\left|a_{i}\right| \leq\left\|\sum_{i=1}^{n} a_{i} X_{i}\right\| \leq(1+\varepsilon) \sum_{i=1}^{n}\left|a_{i}\right| .
$$

Therefore, the sequence $\left(X_{n}\right)_{n \geq 1}$ is equivalent to the canonical basis of $\ell_{1}$.

Theorem 4.2. Let $\omega \leq \alpha \leq \beta<\omega_{1}$ be two countable infinite ordinal numbers. Then $\mathcal{N}(C(\alpha))$ is isomorphic to $\mathcal{N}(C(\beta))$ if, and only if, $\beta<\alpha^{\omega}$.

Proof. For $\alpha \leq \beta<\alpha^{\omega}$ the spaces $C(\alpha)$ and $C(\beta)$ are isomorphic and so $\mathcal{N}(C(\alpha))$ and $\mathcal{N}(C(\beta))$ are also isomorphic. For the converse, suppose that the spaces $\mathcal{N}(C(\alpha))$ and $\mathcal{N}(C(\beta))$ are isomorphic. Then, the space $C(\beta)$ is isomorphic to a subspace of $\mathcal{N}(C(\alpha))=\ell_{1}(\mathbb{N}, C(\alpha))$ and so, by Lemma 4.1. $C(\beta)$ is isomorphic to a subspace of $C(\alpha)$. This implies that $\beta<\alpha^{\omega}$.

\section{REFERENCES}

1. C. Bessaga and A. Pełczyński, Spaces of continuous functions (IV) (On isomorphical classification of spaces of continuous functions), Studia Math. 19 (1960), 53-62. MR0113132 $(22: 3971)$

2. J. Diestel and J.J. Uhl Jr., Vector Measures, Mathematical Surveys 15, Amer. Math. Soc., Providence, RI (1977). MR0453964 (56:12216)

3. A. Grothendieck, Produits tensoriels topologiques et espaces nucléaires. Mem. Amer. Math. Soc. 16 (1955). MR0075539(17:763c)

4. W.B. Johnson, On finite dimensional subspaces of Banach spaces with local unconditional structure. Studia Math. 51 (1974), 225-240. MR0358306 (50:10772)

5. S. Mazurkiewicz and W. Sierpiński, Contributions à la topologie des ensembles dénombrables, Fund. Math. (1) (1920), 17-27.

Centre National de la Recherche Scientifique UMR 6632, Université D'AixMarseille 3, 13397 Marseille Cedex 20, France

E-mail address: christian.samuel@univ-cezanne.fr 